Ann. Sci. forest., 1978, 35 (2), 93-115.

\title{
Recherches sur les biomasses comparées de deux plantations de Pin laricio de Corse avec ou sans fertilisation
}

\author{
J. RANGER \\ Station de Recherches sur les Sols forestiers et la Fertilisation, I.N.R.A., \\ Centre national de Recherches forestières \\ Champenoux, 54280 Seichamps
}

\section{Résumé}

Un protocole d'estimation de biomasses de peuplements forestiers est décrit en détail. Sa complexité est en relation avec l'objectif fixé : préciser l'impact de la fertilisation sur la croissance d'une plantation de Pin laricio de Corse (Pinus nigra Arn. ssp laricio Poiret) en forêt de Moulières (Vienne) et éfudier le cycle des éléments minéraux dans deux ecosystèmes constitués par un traitement fertilisé et le témoin correspondant.

L'étude détaillée par petits compartiments se justifie du fait que la teneur en éléments minéraux est généralement inversement proportionnelle à la biomasse du compartiment.

L'échantillonnage permet une comparaison relative de trois méthodes : stratifiée, de l'arbre moyen et des régressions ; la méthode des régressions semble la plus précise. Elle donne de plus des tarifs simples applicables à des peuplements comparables situés dans des conditions écologiques semblables.

La fertilisation produit un doublement de la production primaire (en y comprenant pour le peuplement fertilisé les produits de l'éclaircie sanitaire réalisée en 1973) de l'origine à 15 ans.

En absolu l'essentiel de l'accroissement de biomasse par la fertilisation se porte sur les troncs.

En valeur relative l'accroissement différentiel sur les principaux compartiments considérés globalement (troncs +100 p. 100 , branches +170 p. 100 et racines +210 p. 100) montre une modification de l'architecture des arbres liée à la fertilisation.

\section{1. - But de l'étude}

Les études de biomasse forestière, florissantes en Belgique wallonne, sont rares en France.

Nous avons profité d'une recherche sur la fertilisation d'une plantation de Pin laricio de Corse (Pinus nigra Arn. ssp. laricio Poiret) pour en mener une à son terme. L'affaire se passe en forêt domaniale de Moulières (Vienne) à une vingtaine de kilomètres au Nord de Poitiers.

II s'agissait de préciser l'impact des engrais (en particulier de leur élément « phosphore ») apportés lors de la plantation 15 ans plus tôt, et d'établir le bilan des éléments nutritifs correspondants.

Deux peuplements sont en comparaison, l'un fertilisé, l'autre pas.

Dans un premier temps - et c'est là le but de cette étude - nous avons effectué une évaluation aussi précise que possible des biomasses ventilées par « comparti- 
ments » (masses foliaires, branches, troncs, racines, que l'on peut subdiviser à leur tour).

Cet article rapporte nos travaux aussi fidèlement que possible dans un domaine sans doute peu connu des lecteurs de cette revue, mais dont une présentation générale à laquelle on pourra se reporter a été faite récemment par J. Pardé (1977).

\section{2. - Présentation écologique des peuplements}

\section{1. - Généralités sur l'essai de Moulières (Vienne)}

L'expérience a été installée par M. Bonneau en 1959. Les résultats initiaux ont été décrits dans deux publications (Bonneau, 1963, 1970).

On a testé l'association d'une fertilisation complète (NPK) à des pratiques culturales diverses (sous solage ou non, culture de lupin, traitement herbicide) sur des sols difficiles à reboiser du fait de leur pauvreté chimique et de leurs propriétés physiques défavorables.

L'expérience comporte trois blocs complets de huit traitements initiaux splités en 1963.

Nous avons comparé sur le bloc III deux traitements : le traitement fertilisé 8.0 (F), dans lequel la fertilisation a été la plus efficace et son témoin le traitement $3.0(T)$.

Traitement $8.0(F)$ : la fertilisation : $92 \mathrm{~kg}$ d'azote, $380 \mathrm{~kg}$ de phosphore $\left(\mathrm{P}_{2} \mathrm{O}_{5}\right)$, $800 \mathrm{~kg}$ de chaux et $160 \mathrm{~kg}$ de potasse à l'hectare enfouis par labour à l'automne 1959. rique).

Plantation : Pin laricio de Corse associé à 1/6 de feuillus (Chêne rouge d'Amé-

Traitement $3.0(T)$ : pas de fertilisation.

Plantation identique à 8.0 après labour.

La plantation de Pin laricio de Corse $1+2$ a été faite à la densité de 4500 plants à l'hectare au printemps 1960.

\section{2. - Le climat général}

C'est un climat atlantique de type intermédiaire entre armoricain et girondin dont les principales caractéristiques sont les suivantes (station météorologique de Chauvigny).

- Pluviométrie annuelle comprise entre 650 et $700 \mathrm{~mm}$ assez bien répartie au cours de l'année.

- Température moyenne annuelle $11^{\circ} 3$ (température moyenne minimale en janvier 308 et moyenne maximale en juillet-août $18^{\circ} 9$ ).

\section{3. - Les sols}

L'expérience est assise sur des sols de type lessivé à pseudogley développés sur une roche mère complexe : argile à meulière du Sannoisien, recouverte d'une couche de limons acides, variable mais peu épaisse.

Il s'agit de sols lourds, mal drainés et pauvres dont quelques caractéristiques sont indiquées dans le tableau 1. 
Les analyses séparées des sols des traitements $F$ et $T$ ont été effectuées sur des prélèvements de 1975 (la granulométrie n'a été déterminée que dans un traitement, vu l'homogénéité des sols).

\section{TABLEAU 1}

Quelques caractéristiques des sols de l'expérience de Moulières

Some characteristics of the soils of the Moulières experiment

\begin{tabular}{|c|c|c|c|c|c|c|c|c|c|c|c|}
\hline \multirow{2}{*}{$\begin{array}{c}\text { Placeau fertilisé } \\
F\end{array}$} & \multirow{2}{*}{ Horizons } & \multirow{2}{*}{$\begin{array}{l}\text { Prof. } \\
\mathrm{cm}\end{array}$} & \multicolumn{3}{|c|}{ Granulométrie } & \multirow{2}{*}{ MO } & \multirow{2}{*}{$N$} & \multirow{2}{*}{$\mathrm{C} / \mathrm{N}$} & \multirow{2}{*}{$\mathrm{pH}$} & \multirow{2}{*}{$\mathrm{P}_{2} \mathrm{O}_{5} *$} & \multirow{2}{*}{$\begin{array}{l}\text { Fer libr } \\
\text { DEB }\end{array}$} \\
\hline & & & A & L & S & & & & & & \\
\hline & $\begin{array}{c}\mathrm{Ap} \\
\mathrm{A}_{2} \text { ef } \mathrm{Bg} \\
\mathrm{Bg} \\
\mathrm{Bg} / \mathrm{C}\end{array}$ & $\left\{\begin{array}{c}0,7 \\
7,15 \\
15,35 \\
35,55 \\
>55\end{array}\right.$ & $\begin{array}{l}14,6 \\
10,3 \\
27 \\
61,3 \\
69,1\end{array}$ & $\begin{array}{r}45,9 \\
45,9 \\
36,7 \\
12,6 \\
8,3\end{array}$ & $\begin{array}{l}39,4 \\
43,8 \\
36,3 \\
26,1 \\
22,6\end{array}$ & $\begin{array}{l}8,68 \\
4,37 \\
2,90 \\
1,00 \\
0,70\end{array}$ & $\begin{array}{l}0,31 \\
0,14 \\
0,13\end{array}$ & $\begin{array}{l}16,3 \\
18,1 \\
17,8\end{array}$ & $\begin{array}{l}4,70 \\
4,25 \\
4,10 \\
4,30 \\
4,40\end{array}$ & $\begin{array}{l}0,11 \\
0,07 \\
0,06 \\
0,04 \\
0,04\end{array}$ & $\begin{array}{l}0,55 \\
0,58 \\
0,65 \\
1,80 \\
2,56\end{array}$ \\
\hline & $\begin{array}{c}\mathrm{Ap} \\
\mathrm{A}_{2} \text { et } \mathrm{Bg} \\
\mathrm{Bg} \\
\mathrm{Bg} / \mathrm{C}\end{array}$ & $\begin{array}{r}0,7 \\
7,15 \\
15,35 \\
35,55 \\
>55\end{array}$ & & & & $\begin{array}{l}5,39 \\
4,11 \\
1,5 \\
0,75 \\
0,7\end{array}$ & $\begin{array}{l}0,22 \\
0,15 \\
0,06\end{array}$ & $\begin{array}{l}14,2 \\
15,9 \\
15,7\end{array}$ & $\begin{array}{l}4,55 \\
3,95 \\
4,00 \\
4,30 \\
4,60\end{array}$ & $\begin{array}{l}0,09 \\
0,05 \\
0,04 \\
0,04 \\
0,04\end{array}$ & $\begin{array}{l}0,68 \\
0,67 \\
1,00 \\
1,9 \\
2,12\end{array}$ \\
\hline
\end{tabular}

Bloc III trait. T. et F. (les données sont exprimées en p. 100 de matière sèche à $105^{\circ} \mathrm{C}$ sauf le Phosphore * qui est exprimé en p. 1000$)$.

\section{4. - La végétation concurrente des arbres}

Dans le placeau fertilisé $F$ le couvert des arbres est fermé et on ne trouve plus, comme végétation concurrente, que quelques ronces et un peu de fougère-aigle.

Dans le placeau témoin T, la lumière arrivant largement au sol autorise le développement d'une végétation concurrente très abondante composée essentiellement de brande (Erica scoparia), d'ajonc nain (Ulex nanus), de callune (Calluna vulgaris), de ronces (Rubus sp.) et de fougère-aigle (Pteris aquilina).

Cette végétation représente une biomasse d'environ $40 \mathrm{t}$ de matière sèche à l'hectare (déterminée par la méthode de la surface unitaire). Nous n'en parlerons pas davantage ici.

\section{5. - Caractéristiques dendrométriques des peuplements}

La surface sur laquelle a été fait l'inventaire dans chacun des traitements est de 0,045 ha. Sur cette surface on fait un inventaire complet du nombre d'arbres (soit environ cent soixante arbres) et on en déduit le nombre d'arbres à l'hectare.

Les mesures de circonférences ne sont faites que sur les trois lignes centrales pour éliminer les effets des traitements voisins (soit quarante arbres dont trente pins). Les caractéristiques dendrométriques sont résumées dans le tableau 2. 
TABLEAU 2

Inventaire des peuplements à l'automne 1975 ; données à l'ha

Stand characteristics (Automn 1975) datas per hectare

Peuplement fertilisé

(F)
Peuplement témoin

(T)

Age de la plantation.

15

Nombre de tiges

- Pin laricio sur pied.....................

- Pin laricio éclaircie sanitaire...............

- Chêne rouge..........................

Total Epicéa .

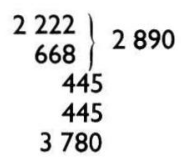

2890

0

222

331

3443

Surface terrière (en $\left.\mathrm{m}^{2} / \mathrm{ha}\right)$

- Pin laricio sur pied.....................

- Pin laricio éclaircie sanitaire...............

- Chêne rouge............................

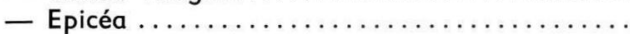

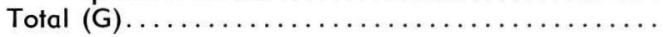

Données relatives au Pin laricio

Diamètre de l'arbre de circonférence moyenne

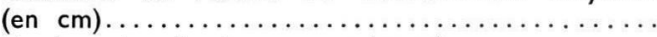
Hauteur de cet arbre moyen (en m).............

Diamètre de l'arbre le plus petit $(e n \mathrm{~cm}) \ldots \ldots \ldots \ldots$.

Diamètre de l'arbre le plus gros $(\mathrm{en} \mathrm{cm}) \ldots \ldots \ldots \ldots$.

29,13

6,28

0,32

0,87

36,61

12,75

7,9

8,6

18,5
18,84

0

0,07

0,26

19,15

L'éclaircie sanitaire signalée dans l'inventaire concerne uniquement le Pin laricio et a été effectuée en 1973 après une attaque parasitaire (vraisemblablement de cronartium) ; ces arbres sont restés au sol. Nous devons les prendre en compte dans notre bilan.

Les épicéas ont été mis en regarni la $2^{\mathrm{e}}$ année. Etant donné le faible pourcentage de surface terrière à l'hectare que représentent les chênes et les épicéas nous n'avons pas déterminé leur biomasse propre. Elle a été assimilée à celle de Pins laricio de même surface terrière, ce qui représente une approximation grossière.

\section{3. - Méthodologie de détermination de la biomasse}

Comme on ne peut pas mesurer arbre par arbre, la biomasse d'un peuplement forestier, il est nécessaire de procéder par échantillonnage.

Le mode de prélèvement choisi est celui de la méthode dite stratifiée (Art et Marks, 1971 ; Satoo 1970) qui consiste à échantillonner l'arbre moyen de chaque classe de circonférence que I'on détermine après l'inventaire complet du peuplement.

Le problème qui se pose est celui de choisir le paramètre définissant l'arbre moyen : hauteur moyenne, diamètre moyen, surface terrière moyenne, volume moyen, etc... De nombreuses études ont montré les variations sur le résultat final, que peut entraîner le choix de l'un ou l'autre de ces paramètres (Baskerville, 1965 ; Crow, 1971).

L'inventaire des peuplements a été effectué avant le prélèvement. On étudie graphiquement la répartition des circonférences dans les deux placeaux. 
Cette distribution est normale, la forme des courbes de Laplace-Gauss étant différente dans les deux traitements

dans

$$
\begin{array}{llll}
\mathrm{F} & \mathrm{m}=40,04 & \text { avec } & \sigma=6,71 \\
\mathrm{~T} & \mathrm{~m}=27,6 & \text { avec } & \sigma=7,64
\end{array}
$$

$\mathrm{m}=$ moyenne arithmétique des circonférences, $\sigma=$ écart type.

Chacun des deux peuplements a été divisé en classes de circonférence $\left(C_{130}\right)$ d'intervalle $6 \mathrm{~cm}$; on élimine pour le prélèvement les classes extrêmes (qui ne contiennent souvent qu'un arbre).

L'arbre échantillonné est, pour chacune des trois classes centrales ainsi définies, l'arbre de circonférence $\left(C_{130}\right)$ moyenne (c'est-à-dire le terme médian de ces classes); soit trois arbres pour chacun des peuplements.

L'arbre moyen de la classe médiane est l'arbre moyen général du placeau (défini sur histogramme).

Les arbres échantillonnés seront appelés arbres de type $A$. Les prélèvements ont été effectués fin octobre, de façon à ce que les compositions foliaires soient stabilisées.

\section{1. - Le prélèvement des échantillons}

\subsection{Les branches.}

Nous avons prélevé quatre branches orientées (N, E, W, S) par verticille * et ceci du bas vers le haut de l'arbre en notant à chaque fois :

- la hauteur relative du verticille dans l'arbre (hr) ;

- la direction de la branche et son diamètre à $5 \mathrm{~cm}$ de l'intersection $\left(D_{5}\right)$ (ceci pour éviter la présence du bourrelet de base, Riedacker 1969) ;

- Ia longueur n'apportant pas de précision améliorée sur le poids (Ottorini, 1974), nous n'avons pas mesuré ce paramètre.

Ces branches sont sectionnées au ras du tronc, immédiatement découpées en pousses annuelles et mises en sacs plastique étanches.

Les branches « supplémentaires" des verticilles échantillonnés sont coupées au ras du tronc et abandonnées sur le terrain. On a au préalable noté leur altitude dans l'arbre (hr) leur orientation et leur diamètre $\left(D_{5}\right)$.

\subsection{La cime.}

Elle est coupée au-dessus de la pousse de l'année $n-3$ et prélevée entièrement ( $n=$ année de prélèvement).

\subsection{Le tronc.}

Il est repéré par sa circonférence à $1,30 \mathrm{~m}$. On le sectionne au ras du sol, on mesure sa hauteur $\mathrm{H}$ et on le divise ensuite en tronçons d'1 $\mathrm{m}$. Chaque billon est numéroté et prélevé totalement.

\subsection{Les racines.}

Le système racinaire de chaque arbre est supposé occuper encore le même volume de sol **. Nous avons donc considéré que ce volume est délimité par le demi-distance le séparant de chacun de ses voisins et ceci sur une profondeur de $60 \mathrm{~cm}$.

Le système racinaire de chaque Pin a été ainsi extrait en séparant les racines en deux classes de diamètre (inf. et sup. à $0,5 \mathrm{~cm}$ de diamètre).

La souche est prélevée séparément.

* Remarque : nous serons amenés à diviser les verticilles en supérieurs et inférieurs (cf. définition sur fig. 1).

** On fait, au prélèvement des racines, une erreur par défaut pour les gros arbres qui sont généralement entourés de petits et occupent un volume plus grand que celui qui leur est attribué. Pour les petits arbres, l'erreur se fait par excès. Il faudrait affecter chaque volume précédemment défini d'un coefficient tenant compte de la taille de l'arbre à déraciner et de son environnement.

Il faut noter qu'en particulier dans le placeau témoin, il est important de séparer les racines de la végétation concurrente de celle des Pins. 


\subsection{Mesures supplémentaires.}

Pour augmenter la précision finale sur l'évaluation de la biomasse des peuplements, nous avons mesuré sept arbres supplémentaires dans chaque placeau, choisis en fonction des fréquences des classes de circonférence. Les classes marginales non échantillonnées sont alors prises en compte.

Sur ces arbres, les paramètres suivants ont été mesurés :

- circonférence à $1,30 \mathrm{~m}\left(\mathrm{C}_{130}\right)$,

- hauteur totale $(\mathrm{H})$,

- altitude des verticilles dans l'arbre (sol = niveau 0$)$,

- diamètre $D_{5}$ et direction de toutes les branches (classées par verticille).

Les arbres ainsi mesurés seront appelés arbres de type $B$.

\section{2. - Mesures au laboratoire}

1. On détermine pour les arbres A le poids humide de tous les compartiments troncs, racines, branches séparées en pousses d'âge différent (les branches ont été divisées en rameaux et feuilles).

2. Divers auteurs ont montré les fluctuations du taux d'humidité au cours d'une journée (Riedacker, 1968 ; Leroy, 1968) et donc les difficultés d'utiliser les poids humides quand on ne peut pas prélever au même instant tous les échantillons.

Pour cette raison, nous préférons raisonner en poids sec. Il faut donc déterminer le taux d'humidité de chaque compartiment. Comme on ne peut mettre six arbres à l'étuve entièrement, on travaille par échantillonnage avec tous les problèmes que cela comporte.

- Pour les feuilles, on procède par «cartage» habituel pour choisir l'aliquote. Nous n'avons mesuré le taux d'humidité que dans une seule direction (Nord), nous avons ensuite vérifié qu'il n'existe pas de différence entre ce taux et celui des feuilles des autres directions en mesurant sur un arbre le taux d'humidité dans les quatre directions.

- Pour les rameaux, le problème est plus délicat, car il faut prendre un échantillon représentatif à la fois du taux d'écorce et de bois et de leurs taux d'humidité respectifs (ces paramètres variant en particulier avec le diamètre des rameaux). Ces prélèvements demandent beaucoup d'attention ef prennent donc beaucoup de temps. C'est pourquoi nous ne l'avons établi précisément que sur une seule direction (en l'occurrence la direction Nord).

Avant d'appliquer les taux d'humidité de l'écorce et du bois des branches Nord aux autres directions, il est nécessaire d'établir la relation qui existe entre eux et ceux des autres directions. A cet effet, nous avons, sur un arbre, mesuré le taux d'humidité dans les quatre directions (NEWS). Nous avons ainsi montré qu'à taux égal d'écorce et de bois, il n'existe pas de différence significative entre le taux d'humidité des branches $\mathrm{N}$ et ceux des autres directions pour des compartiments comparables.

De même pour le taux d'écorce et de bois, on montre qu'il n'y a pas de différence d'architecture entre les branches Nord et celles des autres directions.

- Pour les troncs, on prend comme échantillon une rondelle à la base de chaque billon d'un mètre, à partir de laquelle on mesurera les taux d'écorce et de bois et leur humidité respective.

- Pour les racines, on échantillonne par classe de diamètre (sup. ou inf. à $0,5 \mathrm{~cm}$ ) et on mesure les mêmes paramètres.

Nous avons donc les données de base suivantes :

- Toutes les mesures effectuées sur le terrain.

- Les mesures de laboratoire.

- Pour les feuilles : on connaît le poids humide par âge, par direction et par verticille ; le taux d'humidité mesuré sur les directions Nord que l'on peut appliquer aux autres directions. On a donc facilement la matière sèche par la relation

$$
\text { poids } \sec =\frac{100 \text { poids humide }}{100+\text { taux d'humidité }} \text {. }
$$

- Pour les branches : on connaît le poids total (bois + écorce) par âge, direction et verticille ; on a le taux d'humidité de l'écorce, le taux d'humidité du bois, le pourcentage d'écorce et de bois en poids humide et en poids sec sur les branches Nord (par âge, et verticille). On ne peut donc connaître que le poids de matière sèche d'écorce et de bois pour les directions Nord.

- Pour les troncs et racines : on connaît le poids humide, les pourcentages d'écorce et de bois ef le taux d'humidité de l'écorce et du bois: donc pas de problème pour passer à la matière sèche. 


\section{3. - Principales étapes du calcul de la biomasse des arbres échantillonnés et du calcul de la biomasse du peuplement}

Le problème consiste à rechercher les lois mathématiques qui lient le mieux les paramètres macroscopiques les plus facilement mesurables $\left(D_{5}\right.$, Circ, 1,30 , hauteur, surface terrière, etc...) aux données moins faciles à mesurer (matière sèche par ex.), ceci à partir des arbres échantillonnés (type A). On applique ensuite ces lois aux arbres mesurés (type B) ef l'on obtient ensuite la biomasse à l'hectare d'un tel peuplement.

Une méthode, que d'aucuns pourraient trouver plus rapide, consiste à établir la biomasse de chaque arbre échantillonné, à partir des mesures précitées et de multiplier le résultat de l'arbre moyen de chaque classe par le nombre d'arbres de cette classe : c'est même d'ailleurs la suite logique de la méthode stratifiée (Satoo, 1970).

Mais il se pose d'abord le problème des classes extrêmes, de fréquence peu élevée ; elles interviennent d'une façon non négligeable dans la biomasse. D'autre part, l'établissement de tarifs que l'on pourra appliquer aux arbres mesurés permet de tenir compte de l'architecture d'un plus grand nombre d'arbres et donc d'augmenter la précision sur la biomasse du peuplement.

L'établissement de tarifs permet, en outre, d'aborder les problèmes individuels de croissance et d'architecture comparés des arbres des deux traitements (sans prélèvements supplémentaires). Ils permettent enfin d'appliquer les lois simples finales à des peuplements comparables.

- Plusieurs problèmes se posent quand on veut établir une loi permettant de calculer la biomasse à partir de paramètres simples :

- choix du ou des paramètres à considérer ;

- choix du modèle mathématique qui s'ajuste le mieux au nuage de points expérimentaux.

De très nombreux auteurs se sont penchés sur ce problème : Baskerville, 1965 ; Crow, 1971 ; Egunjobi, 1976 ; Horsiberger, 1969 ; Keays, 1971 ; Kestemont, 1975 ; Magdwick, 1970 ; Mälkönen, 1974 ; Ovington et al., 1967 ; Riedacker, 1968, 1969, 1971 ; Satoo, 1967, 1970 ; Whitaker et Woodwell, 1968, 1969 ; Young, 1971.

Nous avons tiré beaucoup d'enseignements de ces différents travaux mais on constate qu'il n'y a pas de règles universelles, du fait, en particulier, de la diversité des peuplements étudiés et surtout de la diversité des problèmes à résoudre.

D'une façon très générale dans nos équations, la biomasse (exprimée en poids de matière sèche) est la variable dépendante, la variable indépendante étant selon les cas :

- pour les troncs et racines : la circonférence à $1,30 \mathrm{~m}$; branche.

- pour les branches et les feuilles : diamètre à $5 \mathrm{~cm}$ de l'insertion, et hauteur relative de la

Le choix de la circonférence à 1,30 m comme variable indépendante dans l'expression de la biomasse des troncs s'explique par les bons résultats qu'elle permet d'obtenir dans des peuplements simples (équiennes, homogènes, sans concurrence) et par sa facilité de mesure.

Le modèle mathématique choisi au départ a été le plus simple, c'est-à-dire la régression linéaire. On s'aperçoit alors que l'ajustement au nuage de points n'est pas très bon. Il peut être utilisé dans des cas particuliers sur de petits intervalles auxquels il est impératif de se cantonner.

Nous lui avons préféré le modèle dit allométrique largement utilisé en biologie et reconnu depuis longtemps (Huxley et Tessier, 1936, in Lemoine, 1969) comme traduisant le mieux les lois biologiques.

La fonction entre une variable dépendante $Y$ et une variable indépendante $X$ est de la forme :

$$
Y=A X^{B} \text {. }
$$

Les données initiales ont été transformées en log décimaux, d'où

$$
\log Y=A^{\prime}+B \log X \text {. }
$$

L'avantage de cette dernière fonction est sa représentation linéaire en échelle log-log.

II semble également important de préciser qu'il est prudent d'appliquer ces relations pour l'intervalle dans lequel elles ont été calculées.

Nous avons donc :

- pour les troncs:

$$
\begin{aligned}
& \log M S \text { écorce }=a+b \log C_{130} \\
& \log M S \text { bois }=a+b \log C_{130}
\end{aligned}
$$


— pour les racines les équations sont établies par classe de diamètre (sup. ou inf. à $0,5 \mathrm{~cm}$ de diamètre).

La meilleure corrélation a été obłenue en reliant la matière sèche dans son ensemble, à la circonférence de l'arbre à $1,30 \mathrm{~m}\left(\mathrm{C}_{130}\right)$. On applique ensuite un taux moyen d'écorce et de bois

$$
\log M S \text { totale des racines }=a+b \log C_{130} ;
$$

- pour les feuilles : les relations sont plus complexes et il faut tenir compte de deux variables indépendantes, le diamètre à $5 \mathrm{~cm}$ de l'insertion $\left(D_{5}\right)$ et la hauteur relative de la branche dans l'arbre (hr), pour mesurer la masse foliaire. Riedacker (1968) souligne l'importance du paramètre hr qui intègre deux facteurs dépendants biologiquement : l'efficacité de la photosynthèse et la nutrition minérale.

L’équation utilisée est la suivante :

$$
\log M S \text { des feuilles }=a+b \log D_{5}
$$

par année et par niveau de stratification verticale de la nappe foliaire.

La photosynthèse et la nutrition minérale sont en rapport avec la quantité de lumière qui arrive à un niveau donné du couvert végétal. L'intensité lumineuse $\mathrm{I} / \mathrm{I}_{0}$ à une certaine profondeur dans la nappe foliaire est donnée par la formule de Monsi et Saeki adaptée par Satoo (1962) :

$$
\mathrm{I} / \mathrm{I}_{0}=\mathrm{e}^{-\mathrm{KF}}
$$

$K$ est un coefficient d'extinction fonction du peuplement ; F correspond au poids de feuilles au-dessus du niveau considéré.

Cette formule montre que la décroissance de l'intensité lumineuse est continue et donc la photosynthèse et la production de biomasse qui en découlent doivent être aussi continues.

En fait, la variabilité dans la distribution des biomasses foliaires (liée à des facteurs plus ou moins aléatoires de type parasitaires ou climatiques par exemple) associée à une certaine erreur de mesures ne nous ont pas permis d'utiliser la régression multiple entre $M S$ et $\left(D_{5}+h r\right)$. La variation théorique se traduit sur le nuage de points par l'existence de seulement deux zones très nettement différenciées dans la couronne (la cime constitue un $3^{e}$ niveau mais est traitée à part).

L'âge des feuilles entre évidemment dans le calcul de leur biomasse.

- pour les branches vivantes : les paramètres mis en jeu sont les mêmes que pour les feuilles. II est nécessaire d'établir au préalable la relation qui existe entre le diamètre * $\left(D_{5}\right)$ d'une branche et son pourcentage d'écorce et de bois en matière sèche, en établissant les relations par âge, des différentes pousses annuelles et par niveau (défini pour les feuilles).

Pour calculer la biomasse d'écorce et de bois en matière sèche des rameaux des autres directions (par âge et par niveau) connaissant leur seul poids humide total, il est nécessaire de calculer leur matière sèche totale à laquelle on appliquera un pourcentage adéquat d'écorce et de bois.

Le calcul utilise le taux d'humidité corrigé en fonction du diamètre $\left(D_{5}\right)$ de la branche en question puisque le taux d'humidité est fonction de ce diamètre.

Détails du calcul :

- On établit tout d'abord les relations suivantes sur les branches Nord (par année et par niveau) :

$$
\begin{aligned}
& \log \text { e p. } 100=a+b \log D_{5} \\
& \log \text { b p. } 100=a+b \log D_{5} .
\end{aligned}
$$

e et b p. 100 sont les pourcentages respectifs d'écorce et de bois exprimés par rapport à la matière sèche totale.

- Il suffit d'appliquer ces tarifs aux autres directions pour avoir les pourcentages d'écorce et de bois de tout allongement annuel.

- Le taux d'humidité moyen ( $\mathrm{TH}$ ) qu'il faut appliquer à la matière humide d'un allongement annuel (pour les directions EWS) pour avoir sa matière sèche totale est calculé de la façon suivante :

$$
\mathrm{TH}=\frac{\text { THe.e p. 100) }+ \text { (THb.b p. 100) }}{100} .
$$

THe et THb étant respectivement les taux d'humidité de l'écorce et du bois mesurés sur la direction Nord.

* Signalons que l'on utilise pour chaque pousse annuelle le diamètre $D_{5}$ mesuré à la base de la branche. II serait plus précis de mesurer le diamètre à la base de chaque allongement annuel, mais cela nécessiterait un important travail supplémentaire. 
- En affectant les pourcentages adéquats d'écorce et de bois, on obtient les poids secs d'écorce et de bois pour le compartiment considéré.

Pour un rameau donné, les poids de bois et d'écorce obtenus seront considérés comme étant les poids « mesurés».

On établit ensuite par âge et par niveau la relation qui existe entre le diamètre $D_{5}$ d'une branche et ses poids d'écorce et de bois exprimés en matière sèche. Cette relation donnera le tarif définitif pour calculer la biomasse des branches des arbres mesurés (type B).

Ces relations sont du type classique :

$\log$ matière sèche écorce $=a+b \log D_{5}$

$\log$ matière sèche bois $=a+b \log D_{5}$.

A partir de ces lois, on calcule la biomasse des branches des arbres échantillonnés. Nous aurons le poids calculé des branches des arbres échantillonnés :

- pour les branches mortes, le calcul est le même ; il n'y a pas cependant de séparation par pousses annuelles ;

- pour les cimes, la variable indépendante choisie a été la circonférence $C_{130}$ de l'arbre. Etant donné la relative dispersion des mesures, nous avons été amenés à faire entrer dans le même tarif les arbres des deux peuplements.

Pour chaque compartiment, pour les arbres échantillonnés, nous pourrons comparer les poids " mesurés » et les poids calculés, pour évaluer l'erreur.

A ce stade de calcul, on a les tarifs pour mesurer la biomasse compartimentée des arbres de type A et B des deux peuplements.

Le tableau 3 récapitule les diverses équations établies et indique leur champ d'action. II faudrait tester l'égalité des coefficients de corrélation (Dagnélie, 1970) pour infirmer ou confirmer les spécificités.

La figure 1 indique les niveaux de stratification du houppier exprimés en hauteur relative.

Dans le tableau 3 ne figurent pas les tarifs pour les feuilles de $3^{e}$ année. Elles étaient en train de tomber et leur chiffre de biomasse ne sera donné qu'à titre indicatif.

\section{4. - Calcul de la biomasse à l'hectare d'un tel peuplement}

Nous avons sommé les résultats de biomasse par compartiment et par placeau pour les dix arbres.

Comme la distribution est normale et que les arbres échantillonnés et mesurés ont été stratifiés sur toutes les classes de diamètre en fonction de leur fréquence, on peut mesurer la biomasse par compartiment et par mètre carré de surface terrière de chacun des peuplements.

Pour avoir la biomasse du peuplement à l'hectare, il suffit de multiplier le résultat par la surface terrière à l'hectare d'un tel peuplement.

\section{Remarques.}

1. Il se pose le problème de la biomasse des feuillus et des épicéas (mis en regarni). Nous l'avons résolu très sommairement. Comme la surface terrière à l'hectare des feuillus et des épicéas est très faible (tabl. 2) l'erreur sur le résultat final l'est aussi.

2. Le peuplement fertilisé a subi en 1973 une éclaircie sanitaire enlevant environ six cent soixante dix arbres à l'hectare. Ces arbres sont restés sur le terrain. Nous les avons pris en compte pour le calcul de la biomasse du peuplement $(F)$ à cause de leur minéralomasse. L'éclaircie en question a enlevé des arbres de toutes les catégories de circonférence. Nous n'avons pas pris en compte leurs feuilles considérant qu'elles l'avaient déjà été dans les prélèvements de litière.

\section{Etablissement d'un tarif définitif pour le calcul de la biomasse d'un tel peuplement.}

Pour calculer facilement arbre par arbre la biomasse totale ou fractionnée d'un tel peuplement, nous avons établi les relations entre le poids en matière sèche de chaque compartiment d'un arbre et sa circonférence à $1,30 \mathrm{~m}\left(\mathrm{C}_{130}\right)$ (tabl. 4).

Ces équations restent toutefois destinées à l'évaluation d'une biomasse très détaillée en vue d'une étude de minéralomasse.

Nous avons également établi un tarif plus simple (tabl. 5) qui ne tient pas compte de la stratification du houppier et qui permet de calculer facilement la biomasse d'un peuplement semblable à partir de l'inventaire des circonférences. 
Corrélations initiales issues des données expérimentales donnant les tarifs pour le calcul de la biomasse compartimentée de dix arbres par peuplement Initial correlations for experimental datas giving tables to calculate the biomass of the various compartments of ten trees per stand

\begin{tabular}{|c|c|c|c|c|c|c|c|}
\hline Niveau & Compartiment & Age & & $\begin{array}{l}\text { rrélations spécifiques } \\
\text { au placeau fertilisé }\end{array}$ & Corrélations mixtes & & $\begin{array}{l}\text { rrélations spécifiques } \\
\text { u placeau témoin }\end{array}$ \\
\hline \multirow[t]{3}{*}{ Cime } & feuilles & $\begin{array}{l}1 \\
2\end{array}$ & & & $\begin{array}{c}Y_{1}=1,26604 X_{1}+0,06829 \\
n=5 \quad r=0,853 * * \\
Y_{1}=0,41968 X_{1}+0,80340 \\
n=5 \quad r=0,452 \mathrm{NS}\end{array}$ & & \\
\hline & écorce & $\begin{array}{l}1 \\
2 \\
3\end{array}$ & & & $\begin{array}{c}Y_{1}=1,90497 X_{1}-1,28096 \\
n=x^{2}=0,904 * * \\
Y_{1}=2,03761 X_{1}-1,53437 \\
Y_{1}=1,89551 X_{1} \frac{5}{r}=0,111393 * * \\
n=4 \quad r=0,636 \mathrm{NS}\end{array}$ & & \\
\hline & bois & $\begin{array}{l}1 \\
2 \\
3\end{array}$ & & & $\begin{array}{c}Y_{1}=2,11359 X_{1}-1,57902 \\
n={ }^{n}=0,936 * * * \\
Y_{1}=2,26742 X_{1}-1,53520 \\
Y_{1}=2,22836 \times X_{1}-1,28568 \\
n=4 \quad r=0,854 *\end{array}$ & & \\
\hline \multirow[t]{3}{*}{ Verticilles supérieurs } & fevilles & $\begin{array}{l}1 \\
2\end{array}$ & $\begin{array}{l}Y= \\
Y=\end{array}$ & $\begin{array}{c}2,13549 \times+1,24934 \\
n=59 r=0,902 * * * * \\
2,00325 \times+0,98239 \\
n=59 \quad r=0,720 * * * *\end{array}$ & & $\begin{array}{l}\mathrm{Y}= \\
\mathrm{Y}=\end{array}$ & $\begin{array}{r}1,95729 x+1,16661 \\
n=43 r=0,867 * * * * \\
1,96178 x+0,93093 \\
n=43 r=0,784 * * * *\end{array}$ \\
\hline & écorce & $\begin{array}{l}1 \\
2 \\
3 \\
4\end{array}$ & & & $\begin{array}{c}Y=1,49501 \times+0,54241 \\
n=90 \quad r=0,645 * * * * \\
Y=1,04465 \times+0,65402 \\
n=90 r=0,552 * * * * \\
Y=0,20636 \times+0,82682 \\
n=34 r=0,157 \text { NS } \\
Y=2,04528 \times+0,80391 \\
n=44 r=0,841 * * * *\end{array}$ & & \\
\hline & bois & $\begin{array}{l}1 \\
2 \\
3 \\
4\end{array}$ & & & $\begin{array}{c}Y=3,39647 \times n-0,07249 \\
n=90 r=0,892 * * * * \\
Y=3,06011 \times+0,24859 \\
n=90 \quad r=0,889 * * * * \\
Y=4,65289 \times 3-0,02701 \\
n=34=0,963 * * * * \\
Y=3,38724 \times+0,61638 \\
n=L 44 \quad r=0,932 * * * *\end{array}$ & & \\
\hline
\end{tabular}



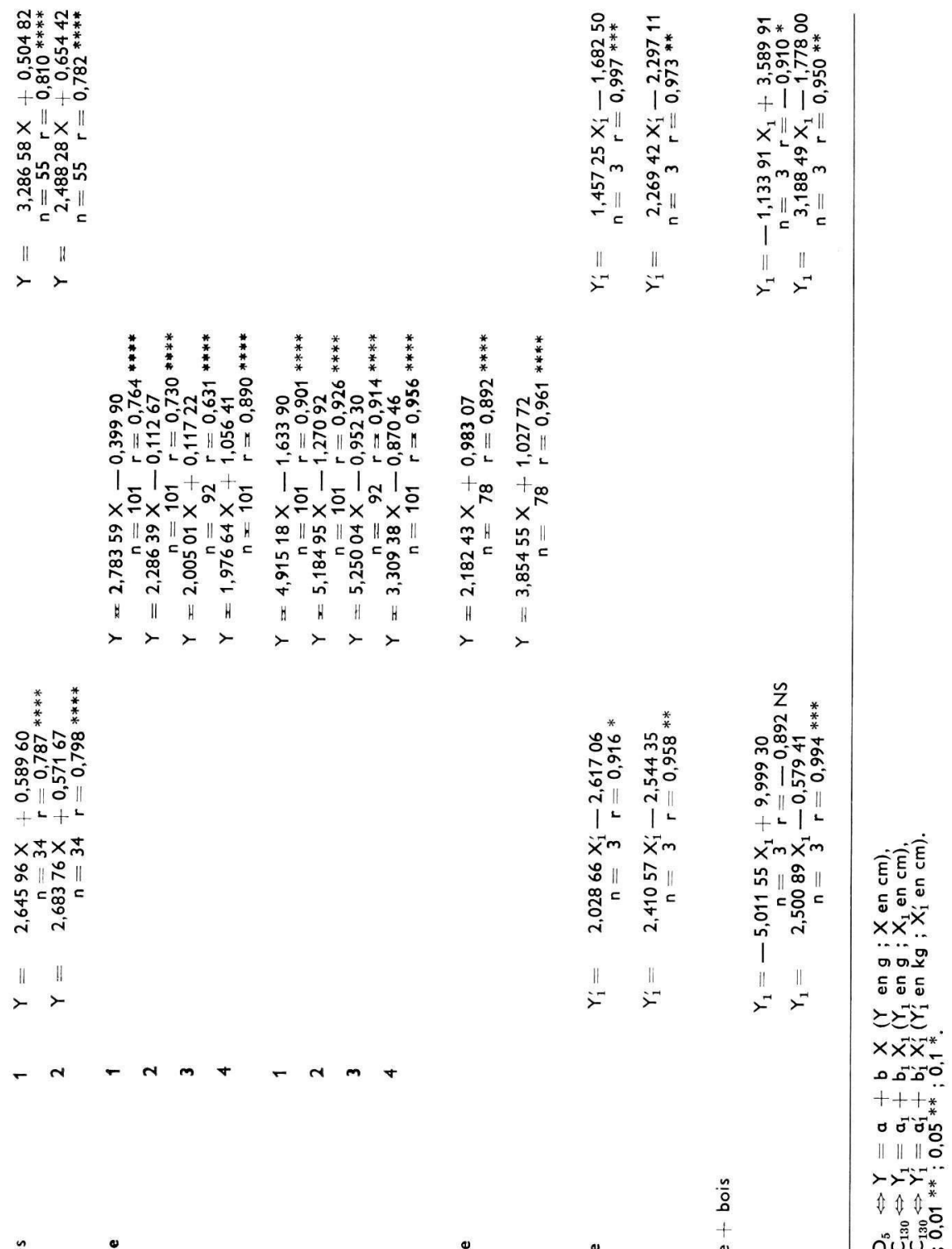

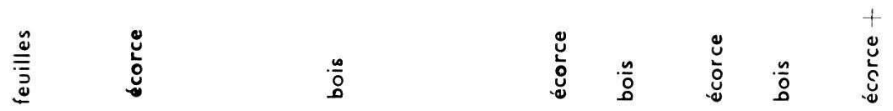

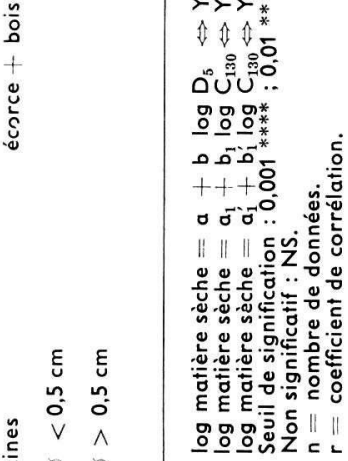


Tableau des corrélations pour les deux peuplements entre la matière sèche d'un " compartiment » et la circonférence de l'arbre à 1,30 m Correlations for the two stands between the dry matter of a compartment and the circumference at $1,30 \mathrm{~m}$

\begin{tabular}{|c|c|c|c|c|c|c|c|c|}
\hline Niveau & Compartiment & $\begin{array}{c}\text { Age } \\
\text { (année) }\end{array}$ & au & $\begin{array}{l}\text { rrélations spécifiques } \\
\text { aceau fertilisé }(n=10)\end{array}$ & Corrélations mixtes $(n=20)$ & & $\begin{array}{l}\text { rélations spécifiques } \\
\text { aceau témoin }(n=10)\end{array}$ & \\
\hline \multirow[t]{3}{*}{ Cime } & feuilles & $\begin{array}{l}1 \\
2\end{array}$ & & & $\begin{array}{l}Y=1,74132 \times-0,63440 \\
Y=2,05659 \times-1,61581\end{array}$ & & & \\
\hline & écorce & $\begin{array}{l}1 \\
2 \\
3\end{array}$ & & & $\begin{array}{l}Y=2,33355 \times-1,91439 \\
Y=2,67098 \times-2,47018 \\
Y=4,69178 \times-5,21565\end{array}$ & & & \\
\hline & bois & $\begin{array}{l}1 \\
2 \\
3\end{array}$ & & & $\begin{array}{l}Y=2,43390 \times-2,02301 \\
Y=2,62995 \times-2,07109 \\
Y=3,05703 \times-2,50184\end{array}$ & & & - \\
\hline \multirow[t]{3}{*}{ Verticilles supérieurs } & feuilles & $\begin{array}{l}1 \\
2\end{array}$ & $\begin{array}{l}Y= \\
Y=\end{array}$ & $\begin{array}{r}2,35886 \times-0,49549 \\
r=0,826^{* * *} \\
2,57723 \times-1,16794 \\
r=0,848^{* * *}\end{array}$ & & $\begin{array}{l}Y= \\
Y=\end{array}$ & $\begin{array}{r}1,78986 X+0,09813 \\
r=0.892^{* * * *} \\
2,11723 x-0,59729 \\
r=0.884^{* * * *}\end{array}$ & $\begin{array}{l}\text { Z } \\
\text { Z } \\
\text { 坚 }\end{array}$ \\
\hline & écorce & $\begin{array}{l}1 \\
2 \\
3 \\
4\end{array}$ & & & $\begin{array}{r}Y=1,27810 X+0,16584 \\
r=0,864 * * * * \\
Y=0,93196 X+0,73039 \\
r=0,831 * * * * \\
Y=0,34567 X+1,53767 \\
r=0,386 * \\
Y=1,43546 X+0,07455 \\
r=0,762 * * * *\end{array}$ & & & \\
\hline & bois & $\begin{array}{l}2 \\
3 \\
4\end{array}$ & & & $\begin{array}{r}Y=2,72263 X-2,25845 \\
r=0,901 * * * * \\
Y=2,45093 X-1,59657 \\
r=0,892 * * * * \\
Y=3,52560 X-3,22709 \\
r=0,893 * * * * \\
Y=2,35407 X-1,19466 \\
r=0,830 * * * *\end{array}$ & & & \\
\hline
\end{tabular}




$\begin{array}{ll}\text { écorce } & 1 \\ 2 \\ 3 \\ 4 \\ \text { bois } & 1 \\ & 2 \\ & 3 \\ & 4\end{array}$

$Y=2,83207 X-2,69658$

$r=0,962 * * * *$
$Y=2,26991 X-1,66815$

$r=0,960 * * * *$

$Y=1,96233 X-1,03647$

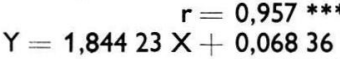

$r=0,950 * * * *$

$Y=5,17619 X-6,93255$

$Y=5,35079 \times-6,75415$

$\begin{aligned} Y=5,35079 & =6,75415 \\ r & =0,947 * * * *\end{aligned}$

$Y=5,49716 X-6,63817$

$Y=3,37380 X+1,62550$

$$
r=0,957 * * * *
$$

Branches mortes

écorce

bois

Troncs + souches

écorce

bois

écorce + bois

$$
\begin{array}{r}
Y=2,12052 \times-0,99303 \\
r=0,793 * * * * \\
Y=3,49211 \times-2,95844 \\
r=0,835 * * * *
\end{array}
$$$$
Y^{\prime}=2,41797 X^{\prime}-3,23376
$$$$
Y^{\prime}=2,62276 X^{\prime}-2,88021
$$

$Y^{\prime}=1,46911 X^{\prime}-1,69919$

$Y^{\prime}=2,39837 X^{\prime}-2,48162$

$$
Y=-6,29226 X+12,02802
$$$$
Y=-1,37714 X+3,93506
$$$$
Y=\quad 3,31249 \times-1,95546
$$

$\log$ matière sèche d'un compartiment $=a,+b \log C_{130} \Leftrightarrow Y Y^{\prime}=a,+b, X^{\prime}(Y$, en g; $X$ en $\mathrm{cm})$

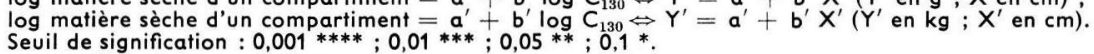

$r=$ coefficient de corrélation 


\section{Remarques.}

Dans les tableaux 4 et 5 , les tarifs pour les cimes, les troncs ef les racines sont issus de données calculées à partir des tarifs du tableau 3 ; il n'y a pas lieu de mettre le coefficient de corrélation qui est évidemment égal à l'unité. Pour les autres compartiments, on utilise les données calculées à partir du tarif initial (tabl. 3), le nouveau calcul se fait par rapport à la variable indépendante $C_{130}$, les fluctuations sont liées au nombre de branches et à leur diamètre pour chacun des dix arbres par placeau entrant dans la corrélation.

\section{5. - Conclusion sur la méthode}

La méthode s'apparente par l'échantillonnage à la méthode dite stratifiée, les calculs se font par la méthode des régressions.

La stratification permet de diminuer l'erreur sur le résultat final (Ovington et al., 1967). Nous tenterons de vérifier cette affirmation.

La complexité de l'échantillonnage, surtout pour les compartiments de faible biomasse, se justifie par le but final du travail : l'étude de la minéralomasse.

\section{4. - Biomasse des peuplements}

\section{1. - Les résultats}

Deux types de données sont à discuter :

- celles concernant l'architecture des arbres, liées aux valeurs individuelles (tabl. 3, 4, 5) ;

- celles concernant la biomasse des peuplements (tabl. 6).

L'intérêt sera porté sur la comparaison des deux peuplements.

On constate globalement que la biomasse des arbres a pratiquement doublé sous l'effet de la fertilisation.

\section{Les troncs.}

L'effet de la fertilisation sur ce comportement est une augmentation de la croissance en hauteur et en diamètre (Bonneau, 1970).

De plus, les arbres des deux traitements ne relèvent pas des mêmes corrélations (pour autant que l'on puisse en juger avec notre échantillonnage). D'après ces droites de régression à diamètre égal, l'arbre du placeau témoin a une biomasse supérieure à celle de l'arbre fertilisé (cette affirmation est toujours vérifiée dans notre intervalle de circonférence ; le point singulier où les droites, issues des équations pour les peuplements $F$ et $T$ se recoupent étant situé à la limite supérieure de l'intervalle en question). Il existe dans notre échantillonnage un arbre de même diamètre dans chaque peuplement qui vérifie cette remarque.

Cette disparité peut être expliquée de deux façons :

- changement du coefficient de forme qui tend à diminuer le volume de l'arbre fertilisé par rapport à l'arbre témoin ;

- changement de densité du bois.

L'arbre fertilisé ayant à diamètre égal une hauteur supérieure, on peut penser que la différence de biomasse est plutôt liée à une diminution de la densité du bois. On retrouve le phénomène observé sur pin maritime (Polge, 1969). 


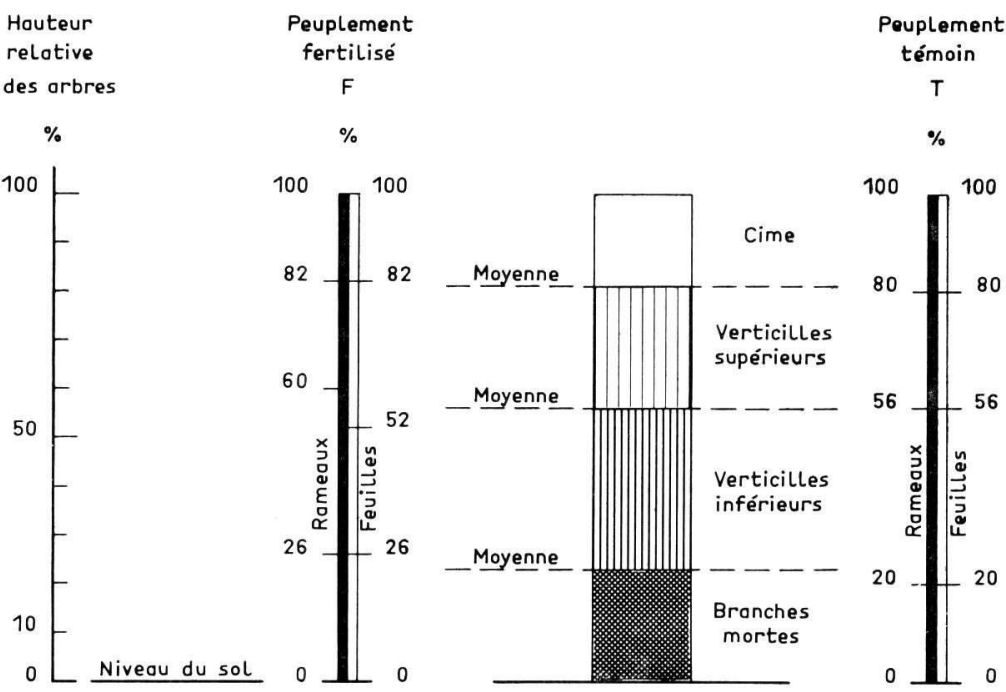

Fig. 1. - Hauteur relative des diverses parties du houppier impliquées darı les tarifs de biomasse des peuplements $F$ et $T$ (tabl. 3 et 4 ) :

- les chiffres de droite concernent la stratification pour les feuilles ;

- les chiffres de gauche concernent la stratification pour les rameaux (écorce et bois);

(les données sont exprimées en pourcentage de la hauteur totale des arbres).

Relative height of various parts of crown involved in biomass fable of stands $F$ and $T$ (tables 3 and 4 ) :

- the numbers on the right relate to the stratification for leaves ;

- the numbers on the left relate to the stratification branches (bark and wood) ;

(datas are expressed in percentage of the total height of trees).

La valeur relative de la biomasse des troncs du peuplement fertilisé par rapport au témoin $\left(\frac{F}{T} \times 100\right)$ est de l'ordre de 200 p. 100.

Les corrélations ont été établies en groupant les troncs et les souches. On a pu estimer que la biomasse des souches représente entre 10 et 15 p. 100 de celle des troncs.

$\mathrm{Si}$ on prend comme densité moyenne du bois la valeur 0,4 , on peut évaluer l'accroissement moyen annuel de chaque peuplement. II serait (sur écorce) de $5,7 \mathrm{~m}^{3} / \mathrm{ha} / \mathrm{an}$ dans le témoin contre $11,4 \mathrm{~m}^{3} /$ ha/an dans le peuplement fertilisé (en ne comptant pas les souches) soit le double.

Les racines.

La différence est très importante entre les deux peuplements.

On retrouve ici l'effet initial de la fertilisation phosphorique qui permet le développement du système racinaire des jeunes plants (Pritchett, 1976).

La biomasse racinaire à l'hectare du peuplement fertilisé est de 310 p. 100 de celle du peuplement témoin. 


\section{TABLEAU 5}

Tableau des tarifs simplifiés pour le calcul de la biomasse des deux peuplements

Simplified tables to calculate the biomass of the two stands

\begin{tabular}{|c|c|c|c|c|c|}
\hline & & Age & Fertilisé & & Témoin \\
\hline \multirow[t]{11}{*}{ BRANCHES } & - Feuilles & 1 & $\begin{array}{c}Y=2,83187 \times-1,04478 \\
n=10 \quad r=0,899 * * * *\end{array}$ & \multicolumn{2}{|c|}{$\begin{array}{c}Y=2,49628 \times-0,65 \\
n=10 \quad r=0,949 * *\end{array}$} \\
\hline & & 2 & $\begin{array}{c}Y=3,46085 \times-2,31676 \\
n=10 \quad r=0,944 * * * *\end{array}$ & \multicolumn{2}{|c|}{$\begin{array}{c}\mathrm{Y}=2,71200 \times-1,126 \\
\mathrm{n}=10 \quad \mathrm{r}=0,825^{* *}\end{array}$} \\
\hline & \multicolumn{5}{|l|}{$\begin{array}{l}\text { - Rameaux } \\
\text { vivants }\end{array}$} \\
\hline & \multirow[t]{4}{*}{ - écorce } & 1 & $Y=1,57687 \times-0,07319$ & $\mathrm{n}=20$ & $\mathbf{r}=0,926 * * * *$ \\
\hline & & 2 & $Y=1,53893 X+0,05561$ & $\mathrm{n}=20$ & $\mathbf{r}=0,964 * * * *$ \\
\hline & & 3 & $Y=1,95834 X-0,46149$ & $\mathrm{n}=20$ & $\mathrm{r}=0,935^{* * * *}$ \\
\hline & & Sup. 3 & $Y=1,92488 X+0,07154$ & $\mathrm{n}=20$ & $\mathrm{r}=0,896^{* * * *}$ \\
\hline & \multirow[t]{4}{*}{ - bois } & 1 & $Y=2,89136 X-2,25101$ & $\mathrm{n}=20$ & $\mathrm{r}=0,963 * * * *$ \\
\hline & & 2 & $Y=2,96329 X-2,08889$ & $\mathrm{n}=20$ & $\mathbf{r}=0,965 * * * *$ \\
\hline & & 3 & $Y=3,83064 X-3,27164$ & $\mathrm{n}=20$ & $\mathrm{r}=0,973 * * * *$ \\
\hline & & Sup. 3 & $Y=3,20283 X-1,75002$ & $\mathrm{n}=20$ & $\mathrm{r}=0,960 * * * *$ \\
\hline
\end{tabular}

- Rameaux

morts

- écorce

$Y=2,12052 \times-0,99303 \quad n=20 \quad r=0,793$

- bois

$Y=3,49211 X-2,95844 \quad n=20 \quad r=0,835$

TRONCS

- écorce

$Y^{\prime}=2,41797 X^{\prime}-3,23376$

$n=10$

$Y^{\prime}=1,46911 X^{\prime}-1,69919$

$Y^{\prime}=2,62276 X^{\prime}-2,88021$

$\mathrm{n}=10$

- bois

$\mathrm{n}=10$

$Y^{\prime}=2,39837 X^{\prime}-2,48162$

$\mathrm{n}=10$

RACINES

$$
\begin{aligned}
& \varnothing<0,5 \mathrm{~cm} \\
& \text { écorce }+ \text { bois } \\
& \varnothing>0,5 \mathrm{~cm} \\
& \text { écorce }+ \text { bois }
\end{aligned}
$$$$
Y=-6,29226 X+12,02802
$$$$
\mathrm{n}=10
$$$$
Y=-1,37714 X+3,93506
$$$$
Y=2,52999 X-0,62533
$$$$
n=10
$$$$
\mathrm{n}=10
$$$$
Y=3,31249 X-1,95546
$$

$\mathrm{n}=10$

$\log$ matière sèche d'un « compartiment» $=a+b \log C_{130} \Leftrightarrow Y=a+b X(Y$ en $g ; X$ en cm) ;

log matière sèche d'un « compartiment» $=a^{\prime}+b^{\prime} \log C_{130} \Leftrightarrow Y^{\prime}=a^{\prime}+b^{\prime} X^{\prime}\left(Y^{\prime}\right.$ en $\mathbf{k g}, X^{\prime}$ en cm). seuil de signification : $0,001 * * * * ; 0,01 * * *$.

$\mathrm{n}=$ nombre de données.

$r=$ coefficient de corrélation. 
TABLEAU 6

Biomasse compartimentée des deux peuplements (les résultats sont exprimés en $\mathrm{kg}$ de matière sèche (à $65^{\circ} \mathrm{C}$ ) à l'ha)

Distribution of total biomass in compartments in the two stands; datas are expressed in $\mathrm{kg}$ of dry matter at $65{ }^{\circ} \mathrm{C}$ per ha

\begin{tabular}{|c|c|c|c|c|c|c|c|c|c|c|c|}
\hline \multirow[b]{2}{*}{ Niveau } & \multirow[b]{2}{*}{ Age } & \multicolumn{5}{|c|}{ Placeau fertilisé $(F)$} & \multicolumn{5}{|c|}{ Placeau témoin (T) } \\
\hline & & feuilles & écorce & bois & $\begin{array}{c}\text { total par } \\
\text { niveau }\end{array}$ & $\begin{array}{c}\text { val. rel. } \\
F / T\end{array}$ & feuilles & écorce & bois & $\begin{array}{l}\text { total par } \\
\text { niveau }\end{array}$ & val. rel. \\
\hline Cime & $\begin{array}{c}1 \\
2 \\
3 \\
\text { s/total }\end{array}$ & $\begin{array}{r}331,3 \\
111,2 \\
37,4 \\
479,9\end{array}$ & $\begin{array}{r}196,1 \\
197,2 \\
821,9 \\
1215,2\end{array}$ & $\begin{array}{r}222,5 \\
428,1 \\
795,7 \\
1436,3\end{array}$ & 3131,4 & 254 & $\begin{array}{r}230,2 \\
72,4 \\
5,5 \\
308,1\end{array}$ & $\begin{array}{r}93,8 \\
86,0 \\
169,2 \\
349,0\end{array}$ & $\begin{array}{l}104,4 \\
185,6 \\
286,1 \\
576,1\end{array}$ & 1233,2 & 100 \\
\hline Verticilles supérieurs & $\begin{array}{c}1 \\
2 \\
3 \\
4 \\
\text { s/total }\end{array}$ & $\begin{array}{r}4956,3 \\
2397,9 \\
413,9 \\
7768,1\end{array}$ & $\begin{array}{r}476,2 \\
472,5 \\
341,6 \\
725,0 \\
2015,3\end{array}$ & $\begin{array}{r}480,7 \\
760,2 \\
1285,8 \\
1362,5 \\
3889,2\end{array}$ & 13672,6 & 267 & $\begin{array}{r}1515,6 \\
955,4 \\
228,7 \\
\\
2699,7\end{array}$ & $\begin{array}{r}286,1 \\
325,5 \\
293,2 \\
407,8 \\
1312,6\end{array}$ & $\begin{array}{r}144,5 \\
263,0 \\
212,8 \\
491,2 \\
1111,5\end{array}$ & 5123,8 & 100 \\
\hline Verticilles inférieurs & $\begin{array}{c}1 \\
2 \\
3 \\
4 \\
\text { s/total }\end{array}$ & $\begin{array}{r}2558,4 \\
2526,9 \\
479,3 \\
5564,6\end{array}$ & $\begin{array}{r}245,8 \\
297,0 \\
390,0 \\
3151,1 \\
4083,9\end{array}$ & $\begin{array}{r}159,9 \\
515,6 \\
1175,9 \\
7940,1 \\
9791,5\end{array}$ & 19440,0 & 290 & $\begin{array}{r}1333,3 \\
1172,9 \\
123,8 \\
2630,0\end{array}$ & $\begin{array}{r}80,4 \\
124,3 \\
187,0 \\
1612,0 \\
2003,7\end{array}$ & $\begin{array}{r}14,5 \\
39,5 \\
83,6 \\
1936,2 \\
2073,8\end{array}$ & 6707,5 & 100 \\
\hline Branches mortes & & & 965,9 & 2273,5 & 3239,4 & 471 & & 310,9 & 376,7 & 687,6 & 100 \\
\hline Total (1) & & 13812,6 & 8280,3 & 17390,5 & 39483,4 & 287 & 5637,8 & 3976,2 & 4138,1 & 13752,1 & 100 \\
\hline \multicolumn{2}{|l|}{ Troncs + souches (2) } & & 13044,8 & 64046,2 & 77091,0 & 197 & & 7747,1 & 31418,7 & 39165,8 & 100 \\
\hline \multicolumn{2}{|c|}{ Biomasse épigée $(3)=(1)+(2)$} & & \multicolumn{2}{|c|}{116574,4} & & 220 & \multicolumn{4}{|c|}{52917,9} & 100 \\
\hline \multicolumn{3}{|l|}{$\begin{array}{l}\text { Racines } \\
\varnothing<0,5 \mathrm{~cm} \\
\varnothing>0,5 \mathrm{~cm} \\
\text { s/total (4) }\end{array}$} & $\begin{array}{r}174,6 \\
1229,4 \\
1404,0\end{array}$ & $\begin{array}{r}334,8 \\
6869,2 \\
7204,0\end{array}$ & 8608,0 & 308,7 & & $\begin{array}{r}70,4 \\
429,1 \\
499,5\end{array}$ & $\begin{array}{r}182,3 \\
2107,0 \\
2289,3\end{array}$ & 2788,8 & 100 \\
\hline \multicolumn{3}{|c|}{ Biomasse totale $(3)+(4)$} & \multicolumn{2}{|c|}{125182,4} & & 225 & \multicolumn{4}{|c|}{55706,7} & 100 \\
\hline
\end{tabular}


Les branches.

Les feuilles.

Les corrélations entre le diamètre d'une branche $D_{5}$ et sa masse foliaire par année et par niveau sont différentes dans les deux traitements. Il y a dans ce cas des tarifs distincts. On constate de plus que dans le placeau fertilisé, la séparation des niveaux pour les feuilles n'est pas exactement la même que pour le placeau témoin. La hauteur relative du niveau inférieur de ce que l'on appelle les verticilles supérieurs est plus basse dans $F$ que dans $T$, d'un verticille. Ce phénomène est en contradiction avec le fait que le couvert du peuplement fertilisé est plus dense que celui du peuplement témoin.

La différence de niveau explique que dans le tableau 3, le nombre de données pour le calcul de la corrélation donnant la biomasse des feuilles des verticilles supérieurs (en sommant $\mathrm{F}$ et $\mathrm{T}$ ) soit supérieur à celui pour le calcul de la biomasse des rameaux de même niveau. La figure 1 précise la remarque précédente.

A âge égal et niveau constant, le poids des feuilles est supérieur dans le peuplement fertilisé. Ceci est lié à deux choses, un nombre différent d'aiguilles par pousse et une taille supérieure des aiguilles des rameaux du placeau fertilisé. La valeur relative de la masse foliaire du peuplement $\mathrm{F}$ par rapport à celle du peuplement T est de 250 p. 100.

\section{Les rameaux.}

II n'y a pas à notre échelle de prospection de différence d'architecture des rameaux pris un à un, entre les deux peuplements. Les mêmes lois régissent les variations de biomasse en fonction du diamètre : il y a tarif unique pour les biomasses « branches » qu'il y ait eu ou non fertilisation. La valeur relative de la biomasse totale des rameaux du peuplement fertilisé par rapport au témoin est de l'ordre de 320 p. 100.

On note une légère augmentation du nombre moyen des branches des arbres fertilisés par rapport aux arbres du témoin ( +4 p. 100).

Si l'on compare non pas le nombre moyen, mais la moyenne des diamètres $\left(D_{5}\right)$ des branches (pour les dix arbres par peuplement), on constate qu'il existe une relation linéaire, commune aux deux peuplements entre cette moyenne et la circonférence de l'arbre à $1,30 \mathrm{~m}$.

La fertilisation augmentant peu le nombre des branches et n'augmentant pas la moyenne du diamètre $\left(D_{5}\right)$ en fonction de la circonférence $\left(C_{130}\right)$ de l'arbre ne fournit pas d'arbres « branchus ».

Au total, la réponse à la fertilisation est très nette (la valeur relative de biomasse $\mathrm{F} / \mathrm{T} \times 100$ est de 230 p. 100) mais assez variable suivant les compartiments. L'architecture des arbres se trouve ainsi modifiée par fertilisation (tabl. 7).

TABLEAU 7

Répartition de la biomasse dans les principaux compartiments exprimée en pourcentage de la biomasse totale Biomass distribution in the main compartments, expressed in percentage of total biomass

\begin{tabular}{lccccc}
\hline \hline & Feuilles & Branches vivantes & Branches mortes & $\begin{array}{c}\text { Troncs et } \\
\text { souches }\end{array}$ & Racines \\
\hline F $\ldots .$. & 11,0 & 17,9 & 2,6 & 61,6 & 6,9 \\
T $\ldots . .6$ & 10,1 & 13,3 & 1,2 & 70,3 & 5,0 \\
\hline \hline
\end{tabular}


On constate que l'essentiel de l'accroissement lié à la fertilisation des peuplements se reporte en valeur absolue sur les troncs et secondairement sur les branches. L'ordre étant différent en valeur relative.

Cependant, si l'étude du nombre de branches et de leur diamètre moyen par arbre montre que les arbres fertilisés ne sont pas spécialement branchus, le tableau 7 indique que le pourcentage de biomasse des branches est nettement supérieur dans le peuplement fertilisé. Ces deux résultats sont contradictoires. En absolu, la biomasse des branches a triplé sous l'effet de la fertilisation.

Les allongements annuels sont supérieurs pour les arbres fertilisés, mais l'architecture des pousses n'étant pas modifiée, la longueur n'intervient pas.

La densité du bois peut être différente. Une partie de la différence de biomasse est expliquée par le nombre de branches supérieur dans $F$ par rapport à $T$. II est cependant difficile d'admettre que les 4 p. 100 supplémentaires suffisent à expliquer le triplement de la biomasse même si le supplément est uniquement dû à des branches de gros diamètres. Il est probable que l'erreur sur les tarifs des petits compartiments (voir § 4.2) aille dans le sens d'une exagération de la différence.

\section{2. - Validité des résultats.}

On peut calculer l'erreur en unités logarithmiques à partir des équations utilisées. D'après Malkonen (1974), on ne peut transformer cette erreur en valeur arithmétique. Il faut alors avoir recours à la formule de Snedecor (in Malkonen, 1974). Ce calcul n'a pas été effectué. Nous avons comparé les données calculées et les données mesurées pour les trois arbres échantillonnés (type A) par peuplement. Pour les valeurs individuelles, les données calculées s'écartent au maximum de \pm 12 p. 100 de la valeur mesurée, alors que sur la somme l'écart n'est que de +1 p. 100 pour le peuplement $T$ et +2 p. 100 pour le peuplement $F$.

Ce chiffre global n'est pas très explicite car les écarts sont d'autant plus importants que la biomasse du compartiment considéré est faible. En fait, l'écart sur ces compartiments intervient peu sur l'écart pondéré.

Globalement pour les deux peuplements, les écarts sont les suivants :

- pour les troncs : inférieur à +1 p. 100 ;

- pour les racines de l'ordre de $+1,5$ p. 100 ;

- pour les branches mortes de -2 à -3 p. 100 ;

- pour la partie non feuillue des branches vivantes de -3 à +10 p. 100 (suivant qu'il s'agisse d'écorces ou de bois) ;

- sur les feuilles, écorce et bois des rameaux de 1 re, $2^{\mathrm{e}}$ et $3^{\mathrm{e}}$ année, l'écart peut atteindre \pm 30 p. 100.

L'écart constaté sur cet échantillonnage stratifié ne traduit qu'une partie de l'erreur sur le peuplement. II faudrait faire intervenir l'erreur d'échantillonnage, la fréquence des classes ef l'erreur sur les mesures.

\section{3. - Comparaison des méthodes}

L'échantillonnage effectué nous permet de comparer la méthode utilisée à la méthode dite de l'arbre moyen, et à la méthode dite stratifiée.

La comparaison de méthodes porte uniquement sur la détermination de la biomasse des Pins laricio des deux peuplements $F$ et $T$ sur pied à 15 ans. 
Si on se reporte à l'inventaire (tabl. 2) sont pris en compte 2222 Pins laricio dans le peuplement $F$ et 2890 dans le peuplement $T$.

Nous établirons les comparaisons par rapport au résultat de ce calcul qui servira de référence.

4.31. Comparaison d la méthode de l'arbre moyen « général » seul mesuré.

L'arbre moyen général du peuplement (dont la répartition des circonférences est gaussienne) est l'arbre moyen de la classe centrale échantillonnée (ce n'est pas l'arbre dont la circonférence est la moyenne arithmétique des circonférences des arbres du peuplement, mais c'est le terme médian de la classe de plus grande fréquence).

II suffit par cette méthode de multiplier la biomasse de cet arbre par le nombre d'arbres pour avoir la biomasse à l'hectare du peuplement.

La valeur de biomasse utilisée pour l'arbre moyen est la valeur mesurée (pour les branches supplémentaires non échantillonnées, nous avons appelé biomasse mesurée la biomasse calculée affectée d'un coefficient qui tient compte de l'écart de la biomasse calculée à la biomasse mesurée de l'arbre en question, en supposant que cet écart est le même pour les valeurs non mesurées).

4.32. Comparaison à la méthode des arbres moyens stratifiés par la classe de circonférence (c'est la méthode dite stratifiée Satoo, 1970).

Pour calculer la biomasse par cette méthode, il suffit de multiplier la biomasse de l'arbre moyen de chaque classe (il s'agit du terme médian de chaque classe) par le nombre d'arbres de la classe en question.

Les classes marginales ont été groupées aux classes échantillonnées les plus proches (leur faible fréquence fait qu'elles interviennent peu, de plus les biais positifs et négatifs se compensent). La valeur de biomasse utilisée pour les arbres moyens de chaque classe est la valeur mesurée (cf. § 3.3) (avec la même correction pour les branches supplémentaires que précédemment).

\subsection{Résultats.}

Ils sont consignés dans le tableau 8.

TABLEAU 8

Comparaison des méthodes

Comparison of methods

\begin{tabular}{lccc}
\hline \hline & $\begin{array}{c}\text { Méthode des régressions } \\
\text { prise comme référence } \\
\text { (notre méthode) }\end{array}$ & $\begin{array}{c}\text { Méthode de l'arbre } \\
\text { moyen « unique » }\end{array}$ & $\begin{array}{c}\text { Méthode stratifiée } \\
\text { par classe de circ. }\end{array}$ \\
\hline $\mathrm{F} \ldots \ldots \ldots$ & 100 & 84,5 & 90 \\
$\mathrm{~T} \ldots \ldots$ & 100 & 75,7 & 101,1 \\
\hline
\end{tabular}

La méthode de l'arbre moyen telle que nous l'avons définie sous-estime le résultat final de 15 à 25 p. 100.

La méthode stratifiée améliore la précision. 
Ces résultats sont conformes à ceux que l'on trouve en bibliographie (Baskerville, 1965 ; Crow, 1967 ; Ovington et al., 1967).

\section{Conclusion}

L'intérêt de la méthode utilisée est de fournir des tarifs relativement précis pour la détermination d'une biomasse ventilée par compartiments en vue d'une étude de minéralomasse.

Le modèle allométrique utilisé permet de calculer la biomasse (exprimée en matière sèche) de chaque compartiment des arbres en fonction de paramètres facilement mesurables.

L'effet de la fertilisation est très net puisque la biomasse a pratiquement doublé dans le traitement $F$ par rapport au traitement $T$. II faut cependant noter que cette biomasse, représentant la production primaire de l'écosystème, inclut les produits enlevés en éclc:rcie sanitaire dans le traitement $F$.

Il est important de constater que l'essentiel du gain de croissance en valeur absolue se porte sur la partie actuellement commercialisable des arbres (les troncs), bien que d'autres compartiments subissent en valeur relative de très forts accroissements.

Les arifs que nous avons pu établir sont valables pour une essence donnée dans un contexte stationnel donné (climat tempéré atlantique, sols chimiquement pauvres et physiquement défavorables).

L'utilisation de ces tarifs pour tout autre peuplement comparable demande un « étalonnage » préalable.

Les résultats obtenus sont difficiles à comparer à d'autres données existantes, comme le montre la récente revue des travaux dans ce domaine, faite par J. Parde (1977).

Reçu pour publication en décembre 1977.

\section{Remerciements}

Je remercie Monsieur J. Parde, directeur de la station de Sylviculture et de Production au CNRF de Nancy, d'avoir bien voulu s'intéresser à ce travail.

Je remercie Messieurs Adrian, Dominique et Pierre Gelhaye, techniciens au CNRF, pour leur collaboration efficace lors de la campagne de prélèvement des échantillons de biomasse. Je remercie ma femme Dominique Ranger pour l'aide précieuse et désintéressée qu'elle m'a apportée pour effectuer les longs calculs et le dépouillement des données.

\section{Summary}

Investigation on compared biomasses of two plantations

of Corsican pine with fertilization or not

\footnotetext{
A biomass estimate method of forest stands is described in details. Its complexity is related to the purpose of the study : to state precisely the fertilization impact on the growth of a Pinus nigra Arn. ssp. Laricio Poiret stand in Moulières (86) (West of France), and to study the nutrient cycle in two ecosystems represented by a fertilized treatment and its check sample.

The amount of mineral elements being generally inversely proportional to the biomass of the compartment justifies the detailed study of small compartments.
} 
Our sampling allows us to make a relative comparison between three methods : stratified, mean tree, and regression estimate. The regression estimate method seems to be the most accurate one. Besides, it gives us simple tables that can be applied to similar stands, put in same ecological situation.

Fertilization doubles the primary production after 15 years (including products of the sanitary clearing made in 1973, amidst fertilized stand).

In absolute value, the main part of biomass increment owing to fertilization is found on the boles. In relative value, the differential increment on main compartments taken as a whole (boles + 100 p. 100 , branches +170 p. 100 and roots +210 p. 100) shows a modification of the architecture of trees owing to fertilization.

\section{Références bibliographiques}

ARTS H. W., MARKS P. L., 1971. A summary table of biomass and net annual primary production in forest ecosystems of the world, pp. 3-32, In " forest biomass studies », XVth IUFRO Congress University of Florida, Gainsville, Florida, USA.

BASKERVILLE G. L., 1975. Estimation of dry weight of tree components and total standing crop in conifer stands. Ecology, 46, 867-869.

BONNEAU M., 1963. Premiers résultats d'une expérience de fertilisation sur Pin laricio de Corse en forêt de Moulières (86). Extrait des ann. de l'Ec. Nat. des Eaux et Forêts et de la St. de Rech. Exp., XX, 3, 314-41.

BONNEAU M., 1970. Résultats après 9 ans de l'essai de fertilisation de Moulières (Vienne). Ann. Sci. Forest., 27 (2), III, 125.

CROW T. R., 1971. Estimation of biomass in an even aged stand. Regression and mean tree techniques. In Forest. biomass słudies, XVth IUFRO Congress University of Florida Gainsville, USA.

DAGNELIE P., 1970. Théorie et méthodes statistiques. 2 volumes presses agronomiques de Gembloux.

DUVIGNEAUD P., 1974. La synthèse écologique. DOIN ed., 296 p.

EGUNJOBI J. K., 1976. An evaluation of five methods for estimating biomass of an even aged plantation of Pinus caribaea L. Oecol. plant., 11 (2), 109-116.

HORISBERGER D., 1969. Etude de la biomasse aérienne du peuplement de Quercus ilex L. de la station du Rouquet. Rapport stage DEA fac. Sci., Montpellier, 27 p.

HUXLEY J. S., TESSIER G., 1936. Zür terminologie des relativen Grössenwachstums. Biol., Zbl., 56, Pp. 381-383.

In LEMOINE B., 1969. Le Pin maritime dans les Landes de Gascogne. Ann. Sci. forest., 26 (4), 445-473.

KEAYS J. L., 1971. Complete tree utilization. An analysis of the litterature. Part I : Unmerchantable top of bole information report UP X. 69. Part II : foliage. Inf. report UP X. 70. Part III : branches. Inf. report UP X. 71. Part IV : Crown and Slash. Inf. report UP X. 77. Part V : Stump, Roots and stump-root system. Inf. report UP X. 79. Forest products laboratory canadian forestry service, depart. of fisheries and forestry, Vancouver, Bristish Columbia.

KESTEMONT P., 1975. Biomasse, nécromasse et productivité aériennes ligneuses de quelques peuplements forestiers en Belgique. Thèse 1 V., 334 p., Fac. de Sciences, Université libre de Bruxelles.

LEROY Ph., 1968. Variations saisonnières des teneurs en eau et éléments minéraux des feuilles de chêne (Quercus pedunculata). Ann. Sci. forest., 25 (2), 83-117.

MAGDWICK H. A. I., 1970. Biomass and productivity models of forest canopies. In Analysis of temperate forest ecosystems (pp. 47-54). Ed. D. E. Reichle.

MÄLKÖNEN E., 1974. Annual primary production and nutrient cycle in some scots pine stands. Metsantutkimuslaitoksen Julkaisusa.

OTTORINI J. M., 1974. Méthode de détermination d'une biomasse foliaire par échantillonnage. Doc. int. station sylv. et Prod. CNRF, no 74/5.

OVINGTON J. D., FORREST W. G., ARMSTRONG J. S., 1967. Tree biomass estimation. In Symposium on primary productivity and mineral cycling in natural ecosystems, PP. 4-31. Uni. Maine Press, ORONO.

PARDE J., 1977. Biomasses forestières et utilisation complète des arbres, RFF.

POLGE H., 1969. Influence de la fertilisation sur la qualité du bois de Pin maritime. Ann. Sci. forest., 26 (1), 45-64. 
PRITCHETT W. L., 1976. Le phosphore dans les sols forestiers. Phosphore ef Agriculture $n^{\circ} 67$, PP. 27-36.

RIEDACKER A., 1968. Méthode d'estimation de la biomasse d'un arbre. D. E. A. Biologie végétale, 31 p., Fac. Sci. Orsay.

RIEDACKER A., 1969. Méthodes indirectes d'estimation de la biomasse des arbres et des peuplements forestiers. Doc. Int. Station Sylv. ef Product. CNRF.

RIEDACKER A., 1971. Measurements of differents components of trees for physiological investigations. In Forest biomass studies, XVth IUFRO Congress University of florida Gainsville, FloridaUSA.

SATOO T., 1970. A synthesis of studies by the harvest method : Primary production. Relations in the Temperate deciduous Forests of Japan. In Analysis of temperate forest Ecosystems. Ed. D. E. Reichle, Pp. 55-72.

WHITAKER R. H., WOODWELL G. M., 1968. Dimension and Production relations of trees and shrubs in the Brookhaven forest. N. Y. J. Ecol., 56, $\mathrm{n}^{\circ}$ 1, 1-25.

WHITAKER R. H., WOODWELL G. M., 1969. Measurement of net primary production of forests. Colloque sur la productivité des Ecosystèmes forestiers, UNESCO PBI Bruxelles, 1969.

YOUNG HE, 1971. Biomass sampling methods for Puckerbrush stands. In Forest Biomass Studies, pp. 179-190. XVth IUFRO Congress University of Florida, Gainsville, USA. 